\title{
ARTIGO CIENTÍEICO \\ Decomposição de esterco bovino e de biomassa de Gliricídia sepium em Neossolo Regolítico
}

\section{Decomposition of bovine esterco and Gliricide sepium biomass in a Regolytic Neosolo}

\author{
Aldrin Martin Pérez-Marin ${ }^{1}$, Maria Iza de Arruda Sarmento ${ }^{2 *}$, Jhony Vendruscolo $^{3}$
}

Resumo: O uso de resíduos orgânicos, como esterco, resíduos de culturas e adubação verde para melhorar a fertilidade do solo e produtividade agrícola é uma das estratégias utilizadas pelos agricultores familiares no Semiárido brasileiro. Nesse contexto, o presente trabalho teve por objetivo avaliar a dinâmica de liberação de N, P e K após a incorporação de esterco bovino ou biomassa de gliricídia (Gliricídia sepium) ao solo. O trabalho foi conduzido em uma área com fileiras de gliricídia espaçadas 6 $\mathrm{m}$ entre si e $1 \mathrm{~m}$ entre as árvores em um Neossolo Regolítico, no Município de Esperança, PB. O delineamento experimental foi em blocos casualizados e parcelas subdivididas, com dois tratamentos principais: presença (CA) ou ausência de aleias de gliricídia (SA), dois tratamentos secundários: adubação com esterco (E) e ramas de gliricídia $(\mathrm{G})$ em quatro repetições. Nas parcelas experimentais foi quantificada a liberação de N, P e K no solo, durante 120 dias, na camada de $0-20 \mathrm{~cm}$. Não houve diferenças significativas das perdas de massa e N, P, K das bolsas de decomposição dos adubos orgânicos entre os sistemas CA e SA. As maiores perdas de N, P e K, ocorreram durante as primeiras duas semanas, sendo mais elevadas no tratamento $\mathrm{G}$ em comparação ao tratamento $\mathrm{E}$, exceto a liberação de $\mathrm{K}$ que teve comportamento similar em ambos os adubos orgânicos. Tanto no sistema SA, como CA, ao longo de todo período de incubação houve mineralização líquida de $\mathrm{N}$, entretanto não foram observados efeitos significativos das adições de gliricídia ou esterco e a testemunha.

Palavras-chave: Adubação orgânica; Incubação de solo; Semiárido brasileiro

\begin{abstract}
The use of organic wastes such as manure, crop residues and green manuring to improve soil fertility and agricultural productivity is one of the strategies used by family farmers in the Brazilian semi-arid. In this context, the objective of this study was to evaluate the dynamics of N, P and K after the incorporation of manure or biomass of gliricídia (Gliricídia sepium). The work was conducted within an area with gliricídia rows spaced $6 \mathrm{~m}$ between each other and $1 \mathrm{~m}$ between trees in a Neossolo Regolítico, in Esperança, PB. The experimental design was a randomized split-plot with two main treatments (with or without gliricídia) and three secondary treatments (fertilization with manure, gliricídia prunnings and control) with four replications. In the experimental plots was measured mineralization of $\mathrm{N}, \mathrm{P}$ and $\mathrm{K}$ in situ in the field for $120 \mathrm{days}$ at $0-20 \mathrm{~cm}$. No significant loss of mass and N, P, K of the decomposition bags of organic fertilizers among the SA and CA systems. The greatest losses of N, P and K occurred during the first two weeks, being higher in the treatment compared to treatment $\mathrm{G}$ E, except that the release of $\mathrm{K}$ had a similar pattern in both organic fertilizers. Both the SA system, such as CA, throughout the incubation period there was net $\mathrm{N}$ mineralization, however there were significant effects of additions of Gliricídia or manure and the control.
\end{abstract}

Key words: Organic fertilization; Incubation of soil; Semiarid brazilian

\footnotetext{
*Autor para correspondência

Recebido para publicação em 29/04/2018; aprovado em 23/09/2018

${ }^{1}$ Pesquisador do Núcleo de Desertificação e Agroecologia do Instituto Nacional do Semiárido e Prof. Permanente do Programa de Pós-Graduação em Ciência do Solo da Universidade Federal da Paraíba, Campus II, Areia, Paraíba.. E-mail: aldrin.perez@insa.gov.br

${ }^{2}$ Graduada em Tecnologia em Agroecologia pelo Instituto Federal da Paraíba, mestranda do Programa de Pós-graduação em Solos e Qualidade de Ecossistemas pela Universidade Federal do Recôncavo da Bahia. E-mail: izasarmento1@gmail.com

${ }^{3}$ Dr. Ciência do Solo pela Universidade Federal da Paraíba, Prof. na Fundação Universidade Federal de Rondônia. E-mail: jhonyvendruscolo@gmail.com
} 


\section{INTRODUÇÃO}

A região semiárida do Brasil compreende uma área de $982.567 \mathrm{~km}^{2}$, abrangendo 1.133 municípios de oito estados da região Nordeste, e o norte do estado de Minas Gerais, na qual reside uma população estimada de 28 milhões de habitantes (IBGE, 2018). Nessa região destaca-se a agricultura de sequeiro com culturas anuais de subsistência, com baixas produtividades decorrentes das irregularidades das chuvas e do manejo inadequado dos solos (PEREZ-MARIN et al., 2017). Em geral, os solos dessa região são deficientes em nitrogênio $(\mathrm{N})$ e fósforo $(\mathrm{P})$, que limitam significativamente a produtividade vegetal (MENEZES et al., 2012). Além disso, o uso de fertilizantes químicos é muito reduzido, devido ao custo e ao risco proporcionado pela variabilidade do regime de chuvas (SILVA, MENEZES, 2007). Nessas condições, a adubação orgânica com esterco animal ou a adubação verde, que incorpore aos solos resíduos de leguminosas arbóreas, são as opções economicamente viáveis para melhorar os níveis de fertilidade nos sistemas de produção de agricultura familiar na região (MENEZES, SALCEDO, 2007).

$O$ esterco é uma fonte alternativa de nutriente amplamente utilizado na região semiárida. No entanto, devido à reduzida disponibilidade de esterco nas propriedades, geralmente os agricultores necessitam comprar esterco de regiões circunvizinhas, o que eleva o custo de produção (GARRIDO et al., 2008, ARAÚJO et al., 2011). Como alternativa a essa problemática, nos últimos anos vem sendo adotada a prática da adubação verde com gliricídia. Planta capaz de melhorar a fertilidade do solo e de aumentar a produtividade das culturas agrícolas associadas, quando usada como adubo-verde (PÉREZ-MARIN et al., 2007; SILVA, MENEZES 2007; CASTRO-RINCON et al., 2018).

Estudos em outras regiões do mundo demonstraram que a incorporação de biomassa de gliricídia, contribuiu para aumentar a produtividade de culturas como milheto e batata, quando comparada com a incorporação de outras leguminosas (MAFONGOYA et al., 2000; BARRETO et al., 2001; COBO et al., 2002; TORMANEN et al., 2018).

No semiárido brasileiro, essa planta vem sendo utilizada em cercas vivas ou cultivada em sistemas de aleias para ser usada como forragem, lenha ou adubo verde, devido à alta qualidade de biomassa (PEREZ-MARIN, et al., 2007) e elevada capacidade de ser inoculada por fixadores de $\mathrm{N}$ atmosférico (BALA et al., 2003) e produzir biomassa em condições de baixa disponibilidade hídrica (PEREZ-MARIN et al., 2007; CHAIN-GUADARRAMA et al., 2018).

Contudo, há necessidade de conhecer a dinâmica de liberação de nutrientes, a partir da mineralização de sua biomassa, visando otimizar a disponibilidade no solo com a demanda pelas culturas e proporcionar estratégias práticas e consistentes para o manejo eficiente do esterco bovino e da biomassa da gliricídia em solos de propriedades de produção agrícola familiar.

Entretanto, na região semiárida existem poucas informações disponíveis que subsidiem o uso eficiente de adubos verdes ou estercos em sistemas de agricultura familiar. Sendo assim, objetivou-se avaliar a dinâmica de liberação de Nitrogênio (N), Fósforo (P) e Potássio (K) no solo, em condições de campo, após a incorporação de esterco bovino ou biomassa de gliricídia a um Neossolo Regolítico no semiárido brasileiro.

\section{MATERIAL E MÉTODOS}

\section{Descrição da área de estudo}

O trabalho foi desenvolvido no Centro Agroecológico São Miguel (CASM), sede da ONG Assessoria e Serviços a Projetos de Agricultura Alternativa (AS-PTA), no município de Esperança, no Agreste paraibano (7¹9'S e 3351'O, 635 $\mathrm{m}$ de altitude). A região apresenta uma estação chuvosa, de março a agosto, e estação seca, de setembro a fevereiro. A precipitação pluviométrica média anual é de $800 \mathrm{~mm}$, e os totais anuais em 2003 e 2004 foram 648 e 1.000 mm, respectivamente. $\mathrm{O}$ solo na área experimental é classificado como Neossolo Regolítico, textura franco-arenosa e com declividade em torno de $5 \%$. As fileiras de gliricídia foram plantadas em 1996, numa área de aproximadamente 0,5 ha, com um espaçamento de $6 \mathrm{~m}$ entre fileiras e $1 \mathrm{~m}$ entre as plantas.

\section{Delineamento experimental e tratamentos}

O delineamento experimental utilizado foi em blocos casualizados em parcelas subdivididas, com quatro repetições. Em 2003, dentro da área experimental, foram demarcadas quatro parcelas de $144 \mathrm{~m}^{2}$ cada $(6 \times 24 \mathrm{~m})$, que corresponderam ao tratamento principal com aleias de gliricídia consorciadas com milho (com aleia - CA). Adjacente a essas parcelas, em áreas sem gliricídia, foram estabelecidas parcelas de mesmo tamanho, nas quais foi cultivado o milho solteiro em sistema convencional (sem aleia - SA). Cada parcela dos tratamentos principais foi subdividida em três subparcelas de $48 \mathrm{~m}^{2}(6 \times 8 \mathrm{~m})$, onde foram implementados os três tratamentos secundários que correspondem a dois tipos de adubação orgânica: 1) aplicação de $15 \mathrm{t} \mathrm{ha}^{-1}$ de massa de matéria seca (aproximadamente $20 \mathrm{t}$ $\mathrm{ha}^{-1}$ de massa de matéria fresca) de esterco bovino (E);2) aplicação de $6,40 \mathrm{t} \mathrm{ha}^{-1}$ de massa de matéria seca (aproximadamente $20 \mathrm{t} \mathrm{ha}^{-1}$ massa de matéria fresca) de gliricídia (folhas e galhos finos fragmentados) (G) e um tratamento testemunha $(\mathrm{T})$, sem adubação orgânica.

$\mathrm{O}$ esterco bovino utilizado no estudo foi obtido em propriedades rurais da região e continha, na matéria seca 9,7; 2,3 e $13 \mathrm{~g} \mathrm{~kg}^{-1}$ de $\mathrm{N}, \mathrm{P}$ e K, respectivamente. A biomassa de gliricídia utilizada, obtida cortando-se pontas de ramas com menos de $1 \mathrm{~cm}$ de diâmetro das plantas presentes na área experimental, continha na matéria seca média de 32, 2,4 e 28 $\mathrm{g} \mathrm{kg}^{-1}$ de $\mathrm{N}, \mathrm{P}$ e $\mathrm{K}$, respectivamente. Os teores médios de matéria seca no esterco e gliricídia utilizados foram 75 e $32 \%$, respectivamente. A biomassa de gliricídia (folhas e galhos finos) foi cortada em pedaços de aproximadamente $10-20 \mathrm{~cm}$ de comprimento para facilitar sua distribuição sobre o solo das parcelas. O esterco e a gliricídia foram incorporados ao solo das parcelas com o auxílio de enxadas até a profundidade de $20 \mathrm{~cm}$, antes do plantio do milho.

Para avaliar a mineralização de nutrientes da biomassa de gliricídia e esterco incorporado ao solo foram conduzidos três experimentos consecutivos in situ nas parcelas experimentais descritas acima, conforme se descrimina a seguir:

\section{Experimento de litter bag após incorporação de adubos orgânicos}

Amostras de esterco (E) e gliricídia (G) foram acondicionadas em bolsas de telas de náilon com dimensões de $10 \times 23 \mathrm{~cm}$ e abertura de $1 \mathrm{~mm}$. A quantidade de material 
colocado nas sacolas dos tratamentos $\mathrm{E}$ e $\mathrm{G}$ no início do ensaio foi de $30 \mathrm{~g}$ com base na matéria fresca, em cada tratamento. As bolsas foram enterradas no solo a aproximadamente $20 \mathrm{~cm}$ de profundidade, simulando a mesma profundidade de incorporação dos adubos orgânicos durante o preparo do solo. Ao todo, foram preparadas 96 bolsas (quatro tratamentos $\mathrm{x}$ quatro repetições $\mathrm{x}$ seis datas de amostragem). A coleta das bolsas de decomposição acorreu aos $0,15,30,45,60,90$ e 120 dias após a data da instalação no campo. Após a coleta, o material vegetal dentro das bolsas foi seco em estufa até peso constante e pesado. Sub-amostras $0,5 \mathrm{~g}$ dos materiais incorporados foram moídas e digeridas pela mistura nítrico-perclórica e analisadas quanto aos teores de $\mathrm{K}$ por fotometria de chama, $\mathrm{P}$ por colorimetria e $\mathrm{N}$ pelo método de Kjeldahl, conforme EMBRAPA (1999). Em outras sub-amostras foi determinado o teor de cinzas do material nas bolsas, para correção do cálculo das perdas de massa e nutrientes do material incorporado, subtraindo-se a massa do solo que aderiu aos materiais colocados dentro das bolsas.

\section{Incubação de solo in situ}

Paralelamente, ao experimento de litter bag após incorporação de adubos orgânicos, realizaram-se três incubações consecutivas de solo in situ, utilizando tubos de alumínio com $20 \mathrm{~cm}$ de comprimento e $5 \mathrm{~cm}$ de diâmetro, abertos no topo e com uma bolsa de resina trocadora de íons no fundo, conforme metodologia descrita por Kolberg et al. (1997). A metodologia consistiu em amostrar o solo das parcelas na camada de $0-20 \mathrm{~cm}$, no início de cada um dos três períodos de incubação $(30,60$ e 90 dias após o plantio do milho), para determinação do conteúdo de $\mathrm{N}-\mathrm{NH}_{4}{ }^{+}$e $\mathrm{N}-\mathrm{NO}_{3}{ }^{-}$. $\mathrm{Na}$ área das duas fileiras centrais de cada parcela foram colocados seis tubos de alumínio para cada período de incubação. Os tubos de $20 \mathrm{~cm}$ foram inseridos no solo e retirados em seguida com auxílio de um alicate; após isso, retirou-se uma camada de $1 \mathrm{~cm}$ de solo da parte inferior do tubo utilizando-se uma espátula e, nesse espaço, colocou-se uma bolsa de malha elástica, contendo $30 \mathrm{~g}$ de uma mistura de resinas trocadoras de cátions e ânions, a qual ficou presa dentro do tubo com auxílio de fita adesiva. Os tubos foram então recolocados no solo e coletados a cada 30 dias de incubação. Imediatamente, após retirar o solo do tubo, uma sub-amostra de $6 \mathrm{~g}$ de solo úmido foi extraída com $\mathrm{KCl} 1 \mathrm{~mol}$ $\mathrm{L}^{-1}$, enquanto que em outra sub-amostra se determinou a umidade do solo. O nitrogênio nas bolsas de resina de cada tubo foi extraído com cinco agitações sucessivas de 16 minutos, com $25 \mathrm{~mL}$ de $\mathrm{KCl} 0,1 \mathrm{~mol} \mathrm{~L}^{-1}$ em cada agitação. A mineralização líquida do solo em cada tubo e cada período foi calculada com ( $\mathrm{N}$ mineral $30 \mathrm{~d}-\mathrm{N}$ mineral inicial) $+\mathrm{N}$ mineral resina. A mineralização de $\mathrm{N}$ no solo de cada parcela e em cada período de incubação foi igual a média da mineralização observada nos seis tubos. As quantidades de N$\mathrm{NH}_{4}{ }^{+}$e $\mathrm{N}-\mathrm{NO}_{3}{ }^{-}$nos extratos de $\mathrm{KCl} 0,1 \mathrm{~mol} \mathrm{~L}^{-1}$ das amostras de solo foram determinadas por colorimetria em um autoanalisador.

\section{Experimento litter bag em superfície com serapilheira de gliricídia}

Durante o período seco (setembro a janeiro) foi coletado o folhedo caído das árvores de gliricídia, utilizando-se cestas com diâmetro de $35 \mathrm{~cm}$ confeccionadas com náilon de abertura de $1 \mathrm{~mm}$ instaladas a uma altura de $20 \mathrm{~cm}$ do solo. $\mathrm{O}$ folhedo coletado foi acondicionado em bolsas de telas de náilon com dimensões $10 \times 10 \mathrm{~cm}$, abertura de $1 \mathrm{~mm} \mathrm{e}$ colocado na superfície do solo ao longo de um transeto: $0 \mathrm{~m}$, $1 \mathrm{~m}$ e $3 \mathrm{~m}$ da fileira de gliricídia. A quantidade de folhedo colocado nas sacolas foi $10 \mathrm{~g}$. Ao todo foram colocadas 144 bolsas ( 3 transetos $\times 4$ repetições $\mathrm{x} 4$ datas de coleta). A coleta das bolsas acorreu aos 30, 60, 90 e 120 dias após a data de colocação no campo. Em seguida o material coletado foi seco em estufa de circulação forçada a $65^{\circ} \mathrm{C}$ por \pm 72 horas, para então ser moído finamente e analisado, conforme descrito acima.

\section{Análises estatísticas}

Os resultados foram tratados estatisticamente por análises de variância, e as médias comparadas pelo teste de Tukey a 5\% de probabilidade.

\section{RESULTADOS E DISCUSSÃO}

\section{Decomposição e liberação de N, P, K após a incorporação de gliricídia e esterco}

Ao longo do período experimental, não houve diferenças significativas entre as perdas de massa e nutrientes das bolsas de decomposição dos adubos orgânicos incorporado no solo nos sistemas de cultivo CA e SA (Figuras 1A-D). Em ambos os sistemas, as maiores perdas de massa seca, N, P e K ocorreram durante as primeiras duas semanas, sendo 35, 38, 12 e $2 \%$, respectivamente, maiores no tratamento $\mathrm{G}$ em comparação ao tratamento E. A liberação de $K$ não apresentou diferenças significativas entre os adubos orgânicos.

Em média, no sistema de cultivo SA, as perdas de massa seca, $\mathrm{N}, \mathrm{P}$ e $\mathrm{K}$ no tratamento $\mathrm{E}$ nos primeiros 15 dias foram 49, 43, 77 e 90\%, respectivamente. As massas e nutrientes restantes aos 120 dias foram 16, 23, 3\% de $\mathrm{N}, \mathrm{P}$ e K, respectivamente, e menos de $1 \%$ de massa seca. Já no tratamento $\mathrm{G}$, foram quantificadas perdas nos primeiros 15 dias de aproximadamente 60, 75, 81 e $90 \%$ de massa seca, N, $\mathrm{P}$ e K, respectivamente (Figuras 1A-D). Após esse período as perdas foram menos intensas e mantiveram-se constantes até o final do período experimental.

No sistema CA as perdas de massa seca, N, P e K nos primeiros 15 dias no tratamento $\mathrm{E}$ foram 43, 31, 73 e $89 \%$ respectivamente, enquanto que no tratamento $G$ as perdas para essas mesmas variáveis foram $65,74,87$ e 94\%, respectivamente (Figuras 1A-D). Na última data de coleta, acima 60 e $85 \%$ da massa seca e nutrientes do tratamento E e $\mathrm{G}$ foram mineralizados, respectivamente.

As maiores perdas de $\mathrm{K}$ pela biomassa de gliricídia e esterco em relação as perdas de $\mathrm{N}$ e $\mathrm{P}$ (Figura 1D), provavelmente, ocorreram devido o $\mathrm{K}$ não fazer parte de nenhum composto orgânico, apresentando alta concentração e baixa retenção no apoplasto (NUNES, et al., 1986; HOLSCHER, et al., 1997; FROUZ, 2018; NAMAGHI et al., 2018). Dessa forma, o K fica prontamente disponível a partir do momento em que o material é incorporado ao solo e inicia a decomposição. 
Figura 1. Percentagem de matéria seca e nutrientes (N, P e K) remanescentes na biomassa de gliricídia (G) e esterco bovino incorporados ao solo a $20 \mathrm{~cm}$ de profundidade aos 15, 30, 45 e 60 e 120 dias em um sistema de cultivo de milho isolado ou em aléias de Gliricídia sepium.
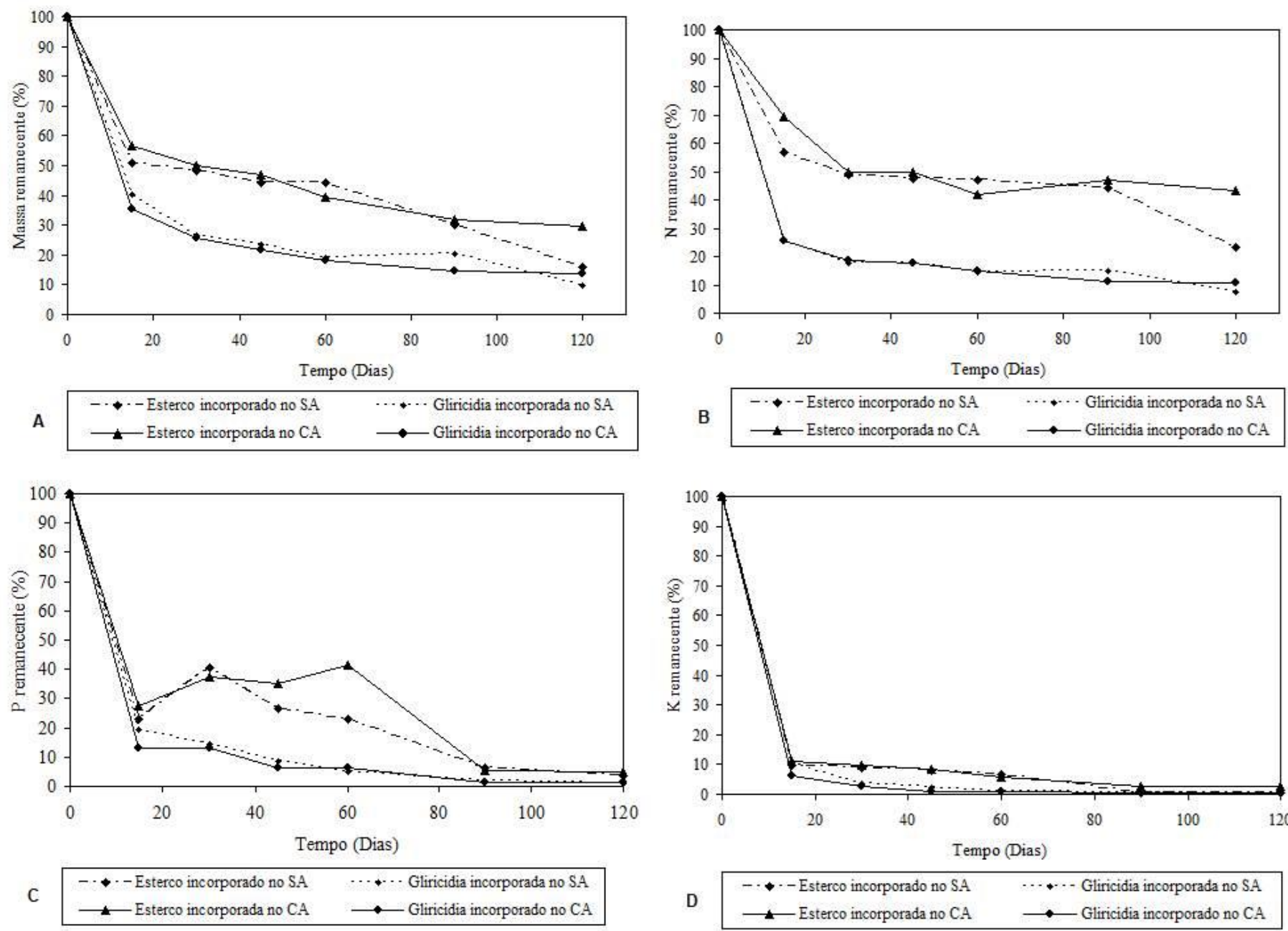

Os resultados mostram que a decomposição e liberação de nutrientes do esterco acontecem de forma mais lenta em relação a da biomassa de gliricídia quando incorporada ao solo, estando de acordo com outros estudos, que avaliaram a decomposição de adubos verdes e esterco (PALM et al., 2001; COBO, et al., 2002; VANLAUWE et al., 2005; SILVA, MENEZES, 2007; CARVALHO et al., 2018; SIERRA et al., 2018). De acordo com Palm et al. (2001), a decomposição mais lenta do esterco, quando comparada a adubos verde e material vegetal, ocorre porque o esterco apresenta menores teores de $\mathrm{N}$ e maiores teores de lignina $\mathrm{e}$ polifenóis do que os adubos verdes, conferindo menor qualidade em relação às outras fontes orgânicas de nutrientes.

\section{Mineralização de $\mathbf{N}$ do solo}

Tanto no sistema sem aleias de gliricídia (SA), como no sistema com aleias (CA), ao longo dos três períodos de incubação e nos três tratamentos de adubação, houve mineralização líquida de $\mathrm{N}$ (Quadro 1). Diferenças significativas de mineralização líquida de $\mathrm{N}$ entre os sistemas de manejo foram constatadas apenas no período de 60 a 90 dias, quando o sistema SA mineralizou mais $\mathrm{N}$ que o sistema CA. Nesse período as parcelas sem árvores mineralizaram cerca de $16 \mathrm{~kg} \mathrm{ha}^{-1}$ a mais de $\mathrm{N}$ em relação as parcelas com

gliricídia. A ausência de significância foi causada principalmente pela variabilidade nas concentrações de $\mathrm{NO}_{3}{ }^{-}$ $\mathrm{N}$ recuperadas na resina, quando ao final de cada tempo de incubação.

Em ambos os sistemas de manejo (SA e CA), as adições de gliricídia ou esterco não apresentaram efeitos significativos sobre a mineralização líquida de N (Quadro 1), embora a mineralização líquida mensal de $\mathrm{N}$ nos tratamentos com gliricídia ou esterco tenha sido mais elevada que a testemunha. De maneira geral, observou-se que ao longo de todo o período experimental o tratamento com gliricídia mineralizou $22 \%$ e $46 \%$ a mais de $\mathrm{N}$, em relação à testemunha, nos sistemas SA e CA, respectivamente. Já a adubação com esterco mineralizou mais N (44\%) no sistema SA em comparação à testemunha, enquanto que no sistema CA foi praticamente igual à testemunha. Menezes e Salcedo (2007), também não encontraram diferenças em N mineralizado entre o controle, sem adições, e parcelas que receberam aplicação de esterco ou gliricídia. Resultados similares também foram descritos por Lupwayi e Haque (1999), onde o N mineralizado não diferiu entre o controle, e parcelas que receberam aplicação de esterco ou ramas de leucena (Leucaena leucocephala) em experimento com milho. 
Quadro 2. Nitrato e amônio extraídos da resina e do solo e, taxa de mineralizado líquida de $\mathrm{N}$ na camada de 0 a $20 \mathrm{~cm}$, em parcelas cultivadas com e sem aleias de Gliricídia sepium, sob dois tipos de adubação orgânica, em Esperança, Paraíba.

\begin{tabular}{|c|c|c|c|c|c|c|c|c|}
\hline \multirow{2}{*}{$\begin{array}{l}\text { Sistema de } \\
\text { Cultivo }\end{array}$} & \multirow{2}{*}{$\begin{array}{c}\text { Adubação } \\
\text { orgânica }\end{array}$} & \multicolumn{2}{|c|}{$\begin{array}{l}\mathrm{N} \text { extraído no solo ao } \\
\text { inicio da incubação }\end{array}$} & \multicolumn{2}{|c|}{$\begin{array}{l}\text { N extraído da resina ao } \\
\text { final da incubação }\end{array}$} & \multicolumn{2}{|c|}{$\begin{array}{l}\mathrm{N} \text { extraído no solo ao } \\
\text { final da incubação }\end{array}$} & \multirow{2}{*}{$\begin{array}{c}\text { Mineralização } \\
\text { líquida de } \mathrm{N}\end{array}$} \\
\hline & & $\mathrm{N}-\mathrm{NO}_{3}{ }^{-}$ & $\mathrm{N}-\mathrm{NH}_{4}^{+}$ & $\mathrm{N}-\mathrm{NO}_{3}^{-}$ & $\begin{array}{l}\mathrm{N}-\mathrm{NH}_{4}^{+} \\
\left.\mathrm{aa}^{-1}\right)\end{array}$ & $\mathrm{N}-\mathrm{NO}_{3}{ }^{-}$ & $\mathrm{N}-\mathrm{NH}_{4}^{+}$ & \\
\hline \multirow{4}{*}{$\begin{array}{l}\text { Sistema sem } \\
\text { aleias (S.A) }\end{array}$} & \multicolumn{8}{|c|}{$\begin{array}{c}\left(\mathrm{kg} \mathrm{ha}^{-1}\right) \\
0 \text { a } 30 \text { dias }\end{array}$} \\
\hline & $\mathrm{T}$ & $19,08 \mathrm{~b}$ & $7,870 \mathrm{a}$ & $22,31 \mathrm{~b}$ & $15,60 \mathrm{a}$ & $35,13 \mathrm{a}$ & $2,25 \mathrm{a}$ & $48,34 \mathrm{a}$ \\
\hline & $\mathrm{G}$ & $53,49 \mathrm{a}$ & $25,84 \mathrm{a}$ & $34,77 \mathrm{a}$ & $22,20 \mathrm{a}$ & $75,50 \mathrm{a}$ & $2,13 \mathrm{a}$ & $55,28 \mathrm{a}$ \\
\hline & $\mathrm{E}$ & $16,66 \mathrm{~b}$ & $11,52 \mathrm{a}$ & $28,75 \mathrm{ab}$ & $38,93 \mathrm{a}$ & $31,39 \mathrm{a}$ & $1,45 \mathrm{a}$ & $72,33 \mathrm{a}$ \\
\hline Média & & $29,74 \mathrm{~A}$ & $15,07^{\mathrm{a}}$ & $28,61 \mathrm{~A}$ & $25,58 \mathrm{~A}$ & $47,34 \mathrm{~A}$ & $1,94 \mathrm{~A}$ & $58,65 \mathrm{~A}$ \\
\hline \multirow{3}{*}{$\begin{array}{l}\text { Sistema com } \\
\text { aleias (C.A) }\end{array}$} & $\mathrm{T}$ & $10,75 \mathrm{~b}$ & 8,60 a & $18,70 \mathrm{~b}$ & $0,75 \mathrm{a}$ & $15,43 \mathrm{a}$ & $2,85 \mathrm{a}$ & $18,38 \mathrm{a}$ \\
\hline & G & $23,00 \mathrm{ab}$ & $22,88 \mathrm{a}$ & 26,98 a & $1,40 \mathrm{a}$ & $38,33 \mathrm{a}$ & $4,28 \mathrm{a}$ & $25,13 \mathrm{a}$ \\
\hline & $\mathrm{E}$ & $24,50 \mathrm{a}$ & $13,90 \mathrm{a}$ & $20,98 \mathrm{ab}$ & $0,78 \mathrm{a}$ & $32,13 \mathrm{a}$ & $2,70 \mathrm{a}$ & $18,18 \mathrm{a}$ \\
\hline Média & & $19,42 \mathrm{~A}$ & $15,13^{\mathrm{a}}$ & $22,22 \mathrm{~A}$ & $0,98 \mathrm{~A}$ & $28,63 \mathrm{~A}$ & $3,28 \mathrm{~A}$ & $20,56 \mathrm{~A}$ \\
\hline \multirow{4}{*}{$\begin{array}{l}\text { Sistema sem } \\
\text { aleias (S.A) }\end{array}$} & \multicolumn{8}{|c|}{30 a 60 dias } \\
\hline & $\mathrm{T}$ & $4,50 \mathrm{~b}$ & $3,25 \mathrm{a}$ & $7,825 \mathrm{a}$ & $9,07 \mathrm{a}$ & $4,25 \mathrm{~b}$ & $3,75 \mathrm{a}$ & $17,15 \mathrm{a}$ \\
\hline & G & $10,50 \mathrm{a}$ & $2,50 \mathrm{a}$ & $11,59 \mathrm{a}$ & $13,35 \mathrm{a}$ & $4,00 \mathrm{~b}$ & $2,75 \mathrm{a}$ & $18,70 \mathrm{a}$ \\
\hline & $\mathrm{E}$ & $6,00 \mathrm{~b}$ & $4,25 \mathrm{a}$ & $10,30 \mathrm{a}$ & $10,20 \mathrm{a}$ & $9,00 \mathrm{a}$ & $4,50 \mathrm{a}$ & $23,75 \mathrm{a}$ \\
\hline Média & & $7,00 \mathrm{~A}$ & $3,33 \mathrm{~B}$ & $9,90 \mathrm{~A}$ & $10,88 \mathrm{~A}$ & $5,75 \mathrm{~A}$ & $3,67 \mathrm{~A}$ & $19,87 \mathrm{~A}$ \\
\hline \multirow{3}{*}{$\begin{array}{l}\text { Sistema com } \\
\text { aleias (C.A) }\end{array}$} & $\mathrm{T}$ & $5,17 \mathrm{a}$ & $8,28 \mathrm{~b}$ & $9,83 \mathrm{c}$ & $0,55 \mathrm{a}$ & $4,05 \quad b$ & $0,08 \mathrm{a}$ & $1,07 \mathrm{a}$ \\
\hline & $\mathrm{G}$ & $17,18 \mathrm{a}$ & $11,98 \mathrm{ab}$ & $25,63 \mathrm{a}$ & $0,55 \mathrm{a}$ & $12,60 \mathrm{a}$ & $0,08 \mathrm{a}$ & $9,73 \mathrm{a}$ \\
\hline & $\mathrm{E}$ & $6,60 \mathrm{a}$ & $13,85 \mathrm{a}$ & $17,25 \mathrm{~b}$ & $0,55 \mathrm{a}$ & $9,13 \mathrm{ab}$ & $0,09 \mathrm{a}$ & $6,58 \mathrm{a}$ \\
\hline Média & & $9,65 \mathrm{~A}$ & $11,37 \mathrm{~A}$ & $17,57 \mathrm{~A} ?$ & $0,55 \mathrm{~B}$ & $8,59 \mathrm{~A}$ & $0,08 \mathrm{~B}$ & $5,79 \mathrm{~A}$ \\
\hline \multirow{4}{*}{$\begin{array}{l}\text { Sistema sem } \\
\text { aleias (S.A) }\end{array}$} & \multicolumn{8}{|c|}{60 a 90 dias } \\
\hline & $\mathrm{T}$ & 3,35 a & $0,30 \mathrm{a}$ & $4,30 \mathrm{~b}$ & $0,36 \mathrm{a}$ & $6,73 \mathrm{a}$ & $4,48 \mathrm{a}$ & $12,20 \mathrm{~b}$ \\
\hline & G & $3,53 \mathrm{a}$ & $1,03 \mathrm{a}$ & $11,68 \mathrm{a}$ & $0,07 \mathrm{a}$ & $11,35 \mathrm{a}$ & $2,45 \mathrm{~b}$ & $21,00 \mathrm{a}$ \\
\hline & $\mathrm{E}$ & $3,78 \mathrm{a}$ & $0,73 \mathrm{a}$ & $8,050 \mathrm{ab}$ & $0,05 \mathrm{a}$ & $9,33 \mathrm{a}$ & $3,08 \mathrm{~b}$ & $16,00 \mathrm{a}$ \\
\hline Média & & $3,55 \mathrm{~A}$ & $0,68 \mathrm{~B}$ & $8,01 \mathrm{~A}$ & $0,16 \mathrm{~A}$ & $9,13 \mathrm{~A}$ & $3,33 \mathrm{~A}$ & $16,40 \mathrm{~A}$ \\
\hline \multirow{3}{*}{$\begin{array}{l}\text { Sistema com } \\
\text { aleias (C.A) }\end{array}$} & $\mathrm{T}$ & $1,78 \mathrm{a}$ & 3,83 a & $7,25 \mathrm{~b}$ & $0,63 \mathrm{a}$ & $3,00 \mathrm{Aa}$ & $2,28 \mathrm{Aa}$ & $7,55 \mathrm{a}$ \\
\hline & G & $2,45 \mathrm{a}$ & 7,18 a & $11,68 \mathrm{a}$ & $0,63 \mathrm{a}$ & $1,88 \mathrm{Ba}$ & $0,88 \mathrm{Ab}$ & $4,53 \mathrm{a}$ \\
\hline & $\mathrm{E}$ & $2,13 \mathrm{a}$ & $6,73 \mathrm{a}$ & $7,45 \mathrm{ab}$ & $0,65 \mathrm{a}$ & $1,40 \mathrm{Ba}$ & $0,55 \mathrm{Ab}$ & $1,70 \mathrm{a}$ \\
\hline Média & & $2,12 \mathrm{~A}$ & $5,91^{\mathrm{a}}$ & $8,79 \mathrm{~A}$ & $0,63 \mathrm{~A}$ & 2,09 & 1,23 & $4,59 \mathrm{~B}$ \\
\hline \multirow{4}{*}{$\begin{array}{l}\text { Sistema sem } \\
\text { aleias (S.A) }\end{array}$} & \multicolumn{8}{|c|}{ Período total (0 a 90 dias) } \\
\hline & $\mathrm{T}$ & $26,93 \mathrm{~b}$ & $11,42 \mathrm{a}$ & $34,44 \mathrm{c}$ & $25,03 \mathrm{a}$ & $46,11 \mathrm{a}$ & $10,47 \mathrm{a}$ & $77,68 \mathrm{a}$ \\
\hline & G & $67,51 \mathrm{a}$ & $29,36 \mathrm{a}$ & 58,03 a & $35,63 \mathrm{a}$ & 90,85 a & $7,33 \mathrm{a}$ & $94,98 \mathrm{a}$ \\
\hline & $\mathrm{E}$ & $26,44 \mathrm{~b}$ & $16,49 \mathrm{a}$ & $47,10 \mathrm{~b}$ & $49,18 \mathrm{a}$ & $49,71 \mathrm{a}$ & $9,03 \mathrm{a}$ & $112,09 \mathrm{a}$ \\
\hline Média & & $40,29 \mathrm{~A}$ & $19,09^{\mathrm{a}}$ & $46,52 \mathrm{~A}$ & $36,61 \mathrm{~A}$ & $62,22 \mathrm{~A}$ & $8,94 \mathrm{~A}$ & $94,92 \mathrm{~A}$ \\
\hline \multirow{3}{*}{$\begin{array}{l}\text { Sistema com } \\
\text { aleias (C.A) }\end{array}$} & $\mathrm{T}$ & $17,69 \mathrm{~b}$ & $20,70 \mathrm{a}$ & $35,78 \mathrm{~b}$ & $1,93 \mathrm{a}$ & $22,48 \mathrm{a}$ & $5,21 \mathrm{a}$ & $27,00 \mathrm{a}$ \\
\hline & G & $42,63 \mathrm{a}$ & $42,03 \mathrm{a}$ & $64,28 \mathrm{a}$ & $2,58 \mathrm{a}$ & $52,81 \mathrm{a}$ & $5,23 \mathrm{a}$ & $39,38 \mathrm{a}$ \\
\hline & $\mathrm{E}$ & $33,23 \mathrm{a}$ & $34,48 \mathrm{a}$ & $45,68 \mathrm{~b}$ & $1,98 \mathrm{a}$ & $42,65 \mathrm{a}$ & $3,34 \mathrm{a}$ & $26,45 \mathrm{a}$ \\
\hline Média & & $31,18 \mathrm{~B}$ & $32,40^{\mathrm{a}}$ & $48,58 \mathrm{~A}$ & $2,16 \mathrm{~B}$ & $39,31 \mathrm{~A}$ & $4,59 \mathrm{~B}$ & $30,94 \mathrm{~A}$ \\
\hline
\end{tabular}

Letras maiúsculas, na coluna, comparam a mineralização de $\mathrm{N}$, entre sistemas de cultivo, e letras minúsculas, na coluna, comparam os tipos de adubação orgânica dentro de cada sistema; letras iguais não diferem entre si pelo teste de Tukey, a 5\% de probabilidade.

A elevada capacidade de mineralização do $\mathrm{N}$ do solo testemunha, associada à variabilidade inerente às medidas de mineralização in situ e a variabilidade pluviométrica resultou em ausência de diferenças significativas na quantidade líquida de $\mathrm{N}$ total mineralizado entre os tratamentos. É possível que essa elevada capacidade de mineralização de $\mathrm{N}$ tenha sido decorrente do pousio nos anos anteriores ao estudo, além da própria decomposição de raízes do milho. Foi constatado que em estudos de incubação em laboratório sob condições controladas de umidade e temperatura, que a incorporação de esterco ou gliricídia tem afetado significativamente a mineralização líquida de $\mathrm{N}$ nos primeiros 50 a 60 dias de incubação (CADISCH et al., 1998; MAFONGOYA et al., 2000; MENEZES; SALCEDO, 2007; SILVA et al., 2007; SIERRA et al., 2018).

O período de incubação de 0 a 30 dias apresentou maior mineralização, tanto em relação às fontes de adubação quanto aos sistemas estudados. Neste período liberou-se 62 e $66 \%$ do total de $\mathrm{N}$ mineralizado dentro do SA e CA, respectivamente (Quadro 1). Os menores valores de mineralização líquida ocorreram nos dois últimos períodos (30 a 60 e 60 a 90 dias). Resultados semelhantes têm sido relatados por Boeira et al., (2001), Silva et al., (2007) e Menezes e Salcedo (2007). Estes autores observaram maior velocidade de mineralização de $\mathrm{N}$ 
no início do período de incubação (64 a 70\%), sendo essa velocidade decrescente com o tempo, além de ser dependente da umidade do solo.

Para este estudo $99 \%$ do $\mathrm{N}$ mineralizado se encontravam na forma de $\mathrm{N}_{-} \mathrm{NO}_{3}{ }^{-}$e o $1 \%$ restante na forma $\mathrm{N}-\mathrm{NH}_{4}{ }^{+}$. Das quantidades totais de $\mathrm{N}_{-} \mathrm{NO}_{3}^{-}$e $\mathrm{N}_{-} \mathrm{NH}_{4}^{+}$mineralizados $75,56 \%$ foram lixiviados para a resina na forma de $\mathrm{N}-\mathrm{NO}_{3}{ }^{-}$, sendo esta lixiviação superior nos tratamentos que receberam adubação com gliricídia, em ambos os sistemas de cultivo e nos três períodos estudados. Uma das causas de maior lixiviação de $\mathrm{NO}_{3}{ }^{-}$para a resina nas parcelas adubadas com gliricídia foi devido o teor de $\mathrm{N}-\mathrm{NO}_{3}-\mathrm{N}$ ter sido superior no solo inicial nas referidas parcelas, e também porque a biomassa de gliricídia apresenta maior facilidade de decomposição do que o esterco bovino, liberando desta forma mais $\mathrm{N}$ em um pequeno espaço de tempo (HANDAYANTO et al., 1997; SANTOS et al., 2010).

\section{Decomposição e liberação de N, P e K da serapilheira de Gliricidia sepium durante estação seca}

Não houve diferenças significativas entre as perdas de massa e nutrientes das bolsas de decomposição colocados em baixo das árvores de $G$. sepium $(0 \mathrm{~m})$ em relação àquelas bolsas colocadas a 1 e $3 \mathrm{~m}$ de distância. Isto se deve principalmente à pouca influência desse sistema de cultivo em aleias sobre o microclima durante a estação seca, devido à característica caducifólia da Gliricidia sepium, que permanece sem folhas durante os períodos de maior estresse hídrico, que associado às podas realizadas ao final das chuvas, ocasiona a permanente redução das copas das árvores e consequente diminuição da capacidade de sombreamento, e, portanto com menor capacidade de influenciar o microclima (MENEZES; SALCEDO, 1999; PEREZ-MARIN et al., 2006).

Figura 2. Percentagem de matéria seca e nutrientes (N, P e K) remanescentes na serapilheira de Gliricidia sepium contidos em bolsas de decomposição, colocadas na superfície do solo e a diferentes distâncias das fileiras de G. sepium, cultivadas em aleias com espaçamento de $6 \mathrm{~m}$ em um Neossolo Regolítico durante a estação seca, em Esperança-PB.
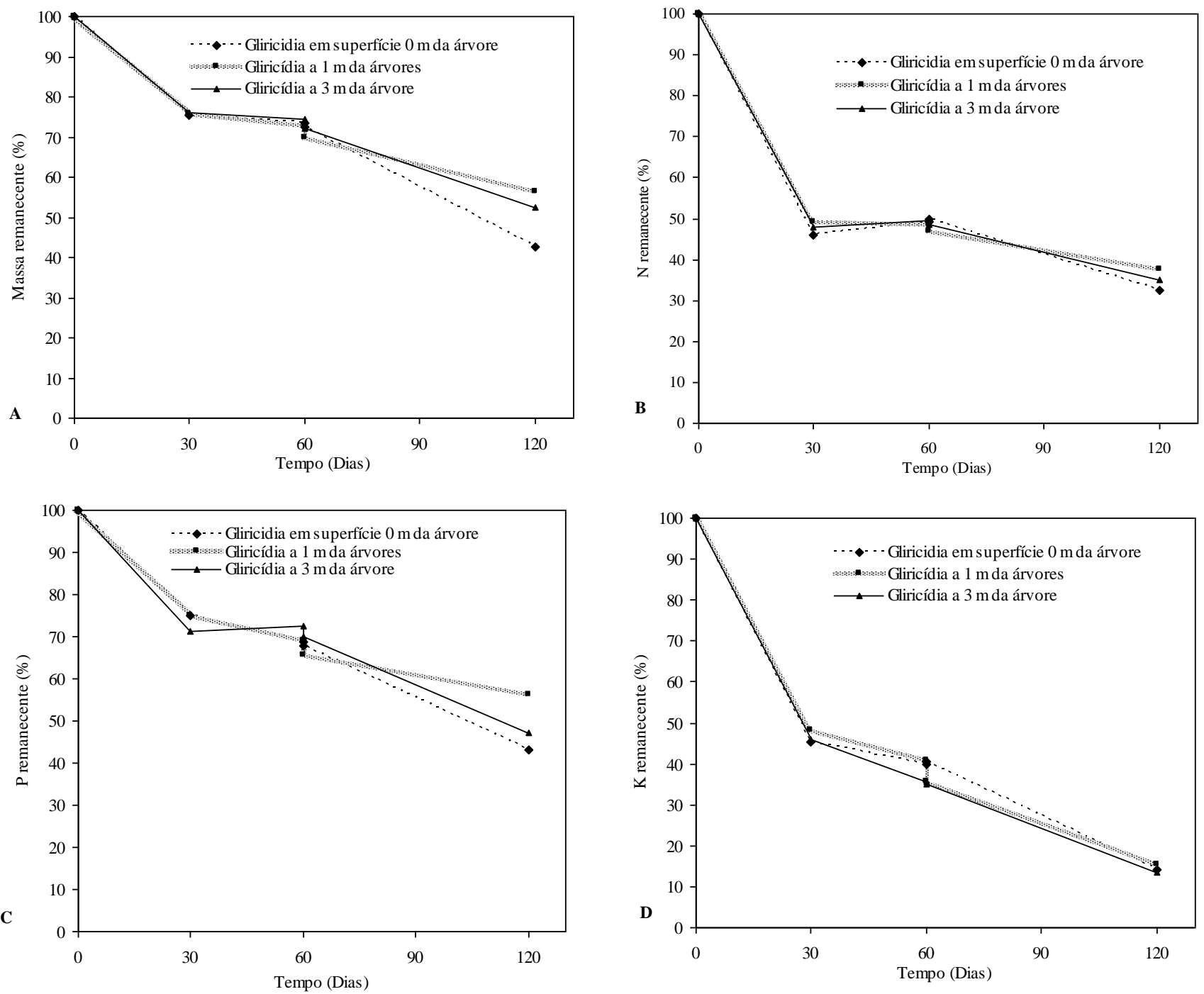

Em geral, as maiores perdas de massa e nutrientes das bolsas de decomposição dispostas na superfície do solo, ocorreram durante as primeiras quatro semanas, variando relativamente pouco ao longo do período avaliado (Figuras 2A e 2D). Em média, nos primeiros 30 dias foram observadas

perdas de aproximadamente $24 \%$ da massa seca. Após esse período, as perdas foram menos intensas e mantiveram-se até o final do período experimental (120 dias), quando foram quantificadas perdas de aproximadamente $49 \%$ da massa seca (Figura 2A). As perdas de N, P e K tiveram comportamento 
semelhante aos de massa seca (Figuras 2B e 2C). Em geral, nos primeiros 30 dias, as perdas de N, P e K foram $52 \%, 26 \%$ e 54\%, respectivamente. Ao final do experimento, $65 \%, 51 \%$ e $86 \%$ do N, P e K foram liberados. Dessa forma, o K fica prontamente disponível para a microbiota, inclusive sendo perdido antes mesmo de o material atingir o solo (FROUZ, 2018). Já os nutrientes associados às moléculas orgânicas como $\mathrm{N}$ e $\mathrm{P}$ são liberados com menor intensidade.

Os resultados sugerem que o plantio de Gliricídia sepium em sistemas de agricultura familiar é uma estratégia para aumentar as entradas de nutrientes através da queda e decomposição da serapilheira e para promover o uso sustentável do solo nos agroecossistemas da região. Entretanto existe demanda de compreender a sincronização da liberação de nutrientes com a demanda pelas culturas em experimentos de campo de longa duração.

\section{CONCLUSÕES}

Em condições de campo não se observou efeito da presença ou ausência de aleias, a mineralização de nutrientes provenientes da adição de gliricídia e esterco bovino, sendo que cerca de $50 \%$ do total de nutrientes mineralizados ocorreram nos primeiros 30 dias de incorporação e incubação no campo.

$\mathrm{O}$ potássio $(\mathrm{K})$ foi o nutriente mais liberado pela serapilheira de G. sepium, seguido de nitrogênio (N) e fósforo (P) as maiores perdas de massa e de nutrientes da serapilheira ocorreram nas quatro primeiras semanas do experimento.

Em ambos os sistemas de manejo com presença (CA) ou ausência de aleias de gliricídia (SA) ao longo de todo o período de incubação houve mineralização líquida de N, sem efeitos significativos sobre a quantidade líquida de $\mathrm{N}$ mineralizado decorrentes da adubação orgânica com esterco e/ou gliricídia e à testemunha.

\section{AGRADECIMENTOS}

Ao CNPq, à ONG Assessoria e Serviços a Projetos em Agricultura Alternativa (AS-PTA), ao Inter American Institute for Global Change Research (IAI) pelo apoio financeiro e à Equipe Técnica da AS-PTA pelo auxílio nas atividades de laboratório e campo.

\section{REFERÊNCIAS}

ARAÚJO, E. R.; DA SILVA' T. O; MENEZES, R. S. C; FRAGA V. S; EVERARDO V. DE S. B. SAMPAIO, E. V. S. B. Biomassa e nutrição mineral de forrageiras cultivadas em solos do semiárido adubados com esterco. Revista Brasileira de Engenharia Agrícola e Ambiental, v.15, 890-895, 2011.

BALA, A.; MURPHY, P.; GILLER, K. E. Distribution and diversity of rhizobia nodulating agroforestry legumes in soil from tree continents in the tropics. Molecular Ecology, n.12, p.917-930, 2003.

BARRETO, A. C.; FERNANDES, F. M. Cultivo de Gliricidia sepium e Leucaena leucocephala em alamedas visando a melhoria dos solos dos tabuleiros costeiros. Pesquisa Agropecuária Brasileira, n.36, p.1287-1293, 2001.
BOEIRA, R. C.; LIGO, M. A. V \& DYNEU, J. F. Mineralização de nitrogênio em solo tropical tratado com lodos de esgoto. Pesquisa Agropecuária Brasileira, n. 11, p.1639-1647, 2001.

CADISCH, G.; HANDAYANTO, E.; MALAMA, C.; SEYNI, F.; GILLER, K. E. Nitrogen recovery from legumes Prunnings and priming effects are governed by the residue quality. Plant and Soil, v.205, p.125-134, 1998.

CARVALHO, D. F.; GOMES, D. P.; NETO, D. H. D.; GUERRA, J. G. M.; ROUWS, J. R. C.; DE OLIVEIRA, P. L. Carrot yield and water-use efficiency under different mulching, organic fertilization and irrigation levels. Revista Brasileira de Engenharia Agrícola e Ambiental, v.22, 445450, 2018.

CASTRO-RINCON, E.; MOJICA-RODRIGUEZ, J. E. Green legume fertilizers: integration in agricultural and livestock systems in the tropics. Agronomia Mesoamericana, v. 29, p. 711-729, 2018.

CHAIN-GUADARRAMA, A.; MARTINEZ-RODRIGUEZ, M.. R.; CARDENAS, J. M.; VILCHEZ-MENDOZA, S.; HARVEY, C. A. Ecosystem-based Adaptation by smallholder basic grain farms in Guatemala and Honduras. Agronomia Mesoamericana, v. 29, 571-583, 2018.

COBO, J. G; BARRIOS, E; KASS, D. C. L; THOMAS, R. J. Decomposition and nutrient release by green manures in a tropical hillside agroecosystem. Plant and soil, n.240, p.331$342,2002$.

EMBRAPA, Empresa Brasileira de Pesquisa Agropecuaria. Informática Agropecuária (Brasília, DF). Manual de análises químicas de solos, plantas e fertilizantes. 1. ed. Brasília, 1999. 370 .

FROUZ, J. Effects of soil macro and mesofauna on litter decomposition and soil organic matter stabilization, Geoderma, v. 332, p. 161-172, 2018.

GARRIDO, M. S.; SAMPAIO, E. V. S. B.; MENEZES, R. S. C. Potencial de adubação orgânica com esterco no Nordeste do Brasil. In: Menezes, R. S. C.; Salcedo, I. H.; Sampaio, E. V. S. B. (Ed.). Fertilidade do solo e produção de biomassa no semi-árido. Recife: UFPE. 2008. p.123-140.

HANDAYANTO, E; CADISCH, G.; GILLER, K. E. Nitrogen release from prunning of legume hedgerow trees in relation to quality of the prunning and incubation method. Plant and Soil, n.60, p.237-248, 1994.

HANDAYANTO, E; GUILLER K. E.; CADISCH, G. Regulating $\mathrm{N}$ release from legume tree pruning by mixing residues of different quality. Soil Biology Biochemistry, 29: 417-1426, 1997.

HOLSCHER, D.; MOLLER, R. F.; DENICH, M.; FOLSTER, H. Nutrient imput-output budget of shifting agriculture in eastern Amazônia. Nutrient Cycling in Agroecosystems, n.47, p.49-57, 1997. 
IBGE. Instituto Brasileiro de Geografia e Estatística. Censo Agropecuário 2017. Rio de Janeiro, 2018. 108 p.

KOLBERG, R. L.; ROUPPET, B.; WESTFALL, D. G.; PETERSON, G. A. Evaluation of an in-situ net soil nitrogen mineralization method in dryland agroecosystems. Soil Science Society of America Journal, Madison, v.61, n.2, p.504-508, 1997.

LUPWAYI, N. Z.; HAQUE, I. Leucaena hedgerow intercropping and cattle manure application in the Ethipian highlands I. Decomposition and nutrient release. Biology and Fertility of Soils, Berlin Heidelberg, v.28, p.182-195, 1999.

MAFONGOYA, P. L.; BARAK, P.; REED, J. D. Carbon, nitrogen and phosphorus mineralization of tree leaves and manure. Biology and Fertility, v.1, p.298-305, 2000.

MENEZES, R. S. C.; SALCEDO, I. H. Mineralização de N após incorporação de adubos orgânicos em um Neossolo Regolítico cultivado com milho. Revista Brasileira de Engenharia Agrícola e Ambiental, Campina Grande, v.11, n.4, p.361-367, 2007.

MENEZES, R. S. C.; SALCEDO, I. H. Influence of tree species on the herbaceous understory and soil chemical characteristics in a silvopastoral system in semi-arid northeastern Brazil. Revista Brasileira de Ciência do Solo, n. 23, p.817-826, 1999.

MENEZES, R. S. C.; SAMPAIO, E. V. S. B.; SILVEIRA, L. M.; TIESSEN, H.; SALCEDO, I. H. Produção de batatinha com incorporação de esterco e/ou crotalária no Agreste paraibano. In: SILVEIRA, L.; PETERSEN, P.; SABOURIN, E., orgs. Agricultura familiar e agroecologia no semi-árido: avanços a partir do agreste da Paraíba. Rio de Janeiro,ASPTA, 2002.

MENEZES, R. S. C.; SAMPAIO E. V. S. B.; GIONGO, V.; PÉREZ-MARIN, A. M. Biogeochemical cycling in terrestrial ecosystems of the Caatinga Biome. Braz. J. Biol, 37 (3):643$653,2012$.

NAMAGHI, M. N.; DAVARYNEJAD, G. H.; ANSARY, H.; NEMATI, H.; FEYZABADY, A. Z. Effects of mulching on soil temperature and moisture variations, leaf nutrient status, growth and yield of pistachio trees (Pistacia vera.L). Scientia Horticulturae, v. 24, p. 115-123, 2018.

NUNES, K. S.; SAMPAIO, E. V. S. B.; ANDRADE, A. G. Ciclagem de nutrientes na mata de dois irmãos através da água de chuva. Cad. Ômega Uni. Fed. PE. Ser. Agron., 2:163174, 1986.

PALM, C. A.; GILlER, K. E.; MAFONGOYA, P. L.; SWIFT, M. J. Management of organic in the tropics: translating theory into practice. Nutrient Cycling in Agroecosystem, v.61, p.63-75, 2001.

PALM, C. A; CATHERINE, N; GACHENGO, DELVE, R. J; CADISCH, G.; GILLER, K. E. Organic inputs for soil fertility management in tropical agroecosystems: aplication of an organic resource database. Agriculture, Ecosystems and Environment, n. 83, p.27-42, 2001.

PEREZ-MARIN, A. M.; MENEZES, R. S. C.; SALCEDO, I. H. Produtividade de milho solteiro ou em aléias de gliricídia adubado com duas fontes orgânicas. Pesq. agropec. bras., Brasília, v.42, n.5, p.669-677, maio 2007.

PEREZ-MARIN, A. M; MENEZES, R. S. C., SAMPAIO, E. V. S. B.; DIAS, E. M. Efeito da gliricídia sepium sobre nutrientes do solo, microclima e produtividade do milho em sistema agroflorestal no agreste paraibano. Revista Brasileira de Ciência do Solo, n. 30, p.555-564, 2006.

SANTOS, A. F. dos; MENEZES, R. S. C.; FRAGA, V. da S.; PEREZ-MARIN, A. M. Efeito residual da adubação orgânica sobre a produtividade de milho em sistema agroflorestal. Revista Brasileira de Engenharia Agrícola e Ambiental. Campina Grande, v. 14, n. 12, p. 1267-1272. 2010.

SIERRA, J.; DESFONTAINES, L. Predicting the in situ rate constant of soil carbon mineralisation from laboratory-based measurements in tropical soils under contrasting tillage management systems. Soil \& Rillage Research, v. 180, p. 175-181, 2018.

SILVA, T. O; MENEZES, R. S. C. Adubação orgânica da batata com esterco e, ou, Crotalaria juncea. II Disponibilidade de N, P e K no solo ao longo do ciclo de cultivo. Revista Brasileira Ciência do Solo, 31:51-61, 2007.

TORMANEN, T.; KITUNEN, V.; LINDROOS, A. J.; HEIKKINEN, J.; SMOLANDER, A. How do logging residues of different tree species affect soil $\mathrm{N}$ cycling after final felling? Forest Ecology and Management, v. 427, p.182$189,2018$.

VANLAUWE, B.; GACHENGO, K.; SHEPHERD, E.; BARRIOS, G; CADISCH, G; PALM, C.A. Laboratory validation of a resource quality-based conceptual framework for organic matter management. Soil Sci. Soc. Am. J, 69:1135-1145, 2005. 